\title{
Temporal-Spatial Analysis of Traffic Congestion Based on Modified CTM
}

\author{
Chenglong Chu, ${ }^{1} \mathrm{Na} \mathrm{Xie}^{2}{ }^{\mathrm{X}}$ iqun Chen, ${ }^{3}$ Yuxin $\mathrm{Wu},{ }^{4}$ and Xiaoxiao Sun ${ }^{5}$ \\ ${ }^{1}$ School of Civil Engineering, Tsinghua University, Beijing 100084, China \\ ${ }^{2}$ School of Management Science and Engineering, Central University of Finance and Economics, Beijing 100081, China \\ ${ }^{3}$ College of Civil Engineering and Architecture, Zhejiang University, Hangzhou 310058, China \\ ${ }^{4}$ School of Accountancy, Central University of Finance and Economics, Beijing 100081, China \\ ${ }^{5}$ School of Statistics and Mathematics, Central University of Finance and Economics, Beijing 100081, China
}

Correspondence should be addressed to Na Xie; xiena@cufe.edu.cn

Received 13 March 2015; Revised 11 May 2015; Accepted 24 May 2015

Academic Editor: Xiaosong Hu

Copyright (C) 2015 Chenglong Chu et al. This is an open access article distributed under the Creative Commons Attribution License, which permits unrestricted use, distribution, and reproduction in any medium, provided the original work is properly cited.

\begin{abstract}
A modified cell transmission model (CTM) is proposed to depict the temporal-spatial evolution of traffic congestion on urban freeways. Specifically, drivers' adaptive behaviors and the corresponding influence on traffic flows are emphasized. Two piecewise linear regression models are proposed to describe the relationship of flow and density (occupancy). Several types of cellular connections are designed to depict urban rapid roads with on/off-ramps and junctions. Based on the data collected on freeway of Queen Elizabeth, Ontario, Canada, we show that the new model provides a relatively higher accuracy of temporal-spatial evolution of traffic congestions.
\end{abstract}

\section{Introduction}

It is well known that traffic flow dynamics estimation is important to transport engineering. To get an accurate estimation, most existing models are based on macroscopic continuum traffic flow modeling. The study of macroscopic continuum traffic flow model began with the LighthillWhitham-Richards (LWR) model independently proposed by Lighthill and Whitham [1] and Richards [2]. The LWR model formulated a hyperbolic partial differential equation (PDE) to describe the temporal-spatial evolution of traffic flow on a homogeneous highway.

Cell transmission model (CTM) is one of convergent numerical approximations to LWR model and was developed by adopting a simplified trapezoidal relationship between traffic flow and traffic density to solve the scalar kinematic wave model $[3,4]$. It assumes a constant free flow speed, $v_{f}$, when density is lower than the critical traffic density and a constant backward shockwave speed, $w$, at a higher density.

Although the linear structure of CTM has the advantage of simplifying traffic control analysis, design, and data-estimation, the assumption of a simplified trapezoidal fundamental diagram seems too strong for general applications. Some more complex models (e.g., Switching Mode Model (SMM)) derived from CTM were presented to reflect the temporal-spatial dynamic features of traffic congestion [5]. Alternatively, the investigation of oscillating congested traffic on freeways based on CTM, Lo et al. [6], leads to a dynamic traffic control formulation designed as dynamic intersection signal control optimization.

It was gradually found that the simple first-order continuum model does not have the ability to explain the amplification of small disturbances on heavy traffic, because no stability condition can be derived from the model [7]. In addition to the simple continuum model, Payne [8] introduced a higher-order model that is derived from car-following model to overcome the shortcomings of LWR model. Based on Payne's model, the deterministic and stochastic finite difference equations of validated high-order model were employed to describe freeway traffic flow dynamics, respectively [912]. Another higher-order continuum model that includes a dynamics equation developed from car-following theory in addition to the conservation equation was introduced 


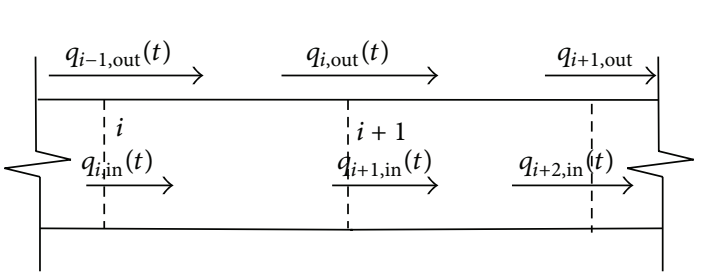

(a)

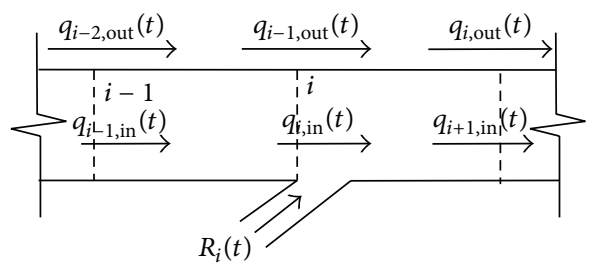

(b)

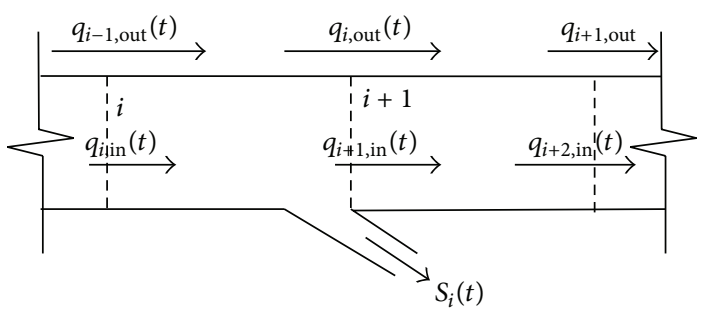

(c)

FIgURE 1: Three typical cell combinations of freeways: (a) with no ramps; (b) with one on-ramp; (c) with one off-ramp.

by Jiang et al. in [7]. This model overcomes the backward travel problem that exists in many other higher-order continuum models and can explain nonlinear dynamical spatialtemporal phenomena on freeways like shock waves, rarefaction waves, stop-and-go waves, and local cluster effects.

The uncertainty of traffic flow also attracts increasing concerns. Alecsandru [13] developed a stochastic compositional model of the evolution of traffic flows on freeways to extend the Daganzo cell transmission model by defining sending and receiving functions explicitly as random variables and by also specifying the dynamics of the average speed in each cell. Differently, an entropy solution was proposed for the LWR model with a flow density relationship of being piecewise quadratic, continuous, and concave, but not differentiable at the junction points where two quadratic polynomials meet [14]. Several alternative models were also developed in [15, $16]$.

In this paper, a modified cell transmission model (CTM) is proposed to depict the temporal-spatial evolution of traffic congestion on urban freeways. Specifically, drivers' adaptive behaviors and the corresponding influence on traffic flows are emphasized. Two piecewise linear regression models are used to describe the relationship of flow and density (occupancy). Several types of cellular connections are designed to depict urban rapid roads with on/off-ramps and junctions.

The rest of this paper is organized as follows. In Section 2, a new formation of CTM is presented. Section 3 discusses two critical issues when linear initialization of density and local perturbation analysis are conducted to reflect the dynamic and nonequilibrium properties of traffic flow. Section 4 addresses the two applications of spatial-temporal evolution analysis based on the proposed method with real detective data on unban freeways. Finally, main conclusions are summarized in Section 5.

\section{An Improved Spatial-Temporal Finite Difference Model}

The LWR model and the selected higher-order continuum model can be written in two PDEs as follows:

$$
\begin{aligned}
& \frac{\partial \rho}{\partial t}+\frac{\partial \rho v}{\partial x}=g(x, t) \\
& \frac{\partial v}{\partial t}+v \frac{\partial v}{\partial x}=\frac{v_{e}(\rho)-v}{T}-c(\rho, v) \frac{\partial p(\rho, v)}{\partial x}
\end{aligned}
$$

where $t$ and $x$ denote time and distance from the origin, $\rho(x, t)$ is the traffic density, $v(x, t)$ is the space mean speed, $g(x, t)$ is net-merging traffic flow, $T$ is the relaxation time, $v_{e}(\rho)$ is the steady-state speed-density relationship implied by car-following model, and $p(\rho, v)$ denotes the traffic pressure from upstream, and $c(\rho, v)$ means the coefficient of traffic pressure.

2.1. Finite Difference of the First-Order Equation. For the firstorder model, CTM is an efficient finite difference solution scheme for realistic freeways and urban road networks. Figure 1 demonstrates three typical cell combinations of freeways, considering on/off-ramps.

For the basic segments without on/off-ramps demonstrated in Figure 1(a), the conservation equation can be derived from LWR model as follows:

$$
\begin{aligned}
\rho_{i}(t+1) & =\rho_{i}(t)+\frac{T_{s}}{\lambda_{i} l_{i}}\left[q_{i-1, i}(t)-q_{i, i+1}(t)\right] \\
& =\rho_{i}(t)+\frac{T_{s}}{\lambda_{i} l_{i}}\left[q_{i, \text { in }}(t)-q_{i, \text { out }}(t)\right],
\end{aligned}
$$

where $i$ denotes the cell number, $T_{s}$ is the time interval, $l_{i}$ is the length of cell $i, \lambda_{i}$ is the lane number of cell $i, \rho_{i}(t)$ means 
the vehicle density of cell $i$ at the time of $t$. And $q_{i \text {,in }}(t)$ and $q_{i \text {,out }}(t)$, respectively, denote the traffic flow entering and leaving cell $i$ during the $t$ th time interval, $T_{s}[t, t+1)$, including flows along the mainline and the on- and off-ramps.

In order to refine the transmission rules, it is assumed that two adjacent cells can be divided into one of five connection modes: (1) "free flow-free flow" (FF), (2) "congestioncongestion" (CC), (3) "congestion free flow" (CF), (4) "free flow congestion 1" (FC1), in which the wave front is moving downstream, and (5) "free flow congestion 2" (FC2), in which the wave front is moving downstream [5]. The SMM is composed of several linear models; straightforward linear techniques for model analysis and control design can be applied to the individual linear subsystems. The wave propagation direction is usually difficult to detect exactly. So, four distinguishable states are formulated in this paper as follows:

FF:

$$
\begin{aligned}
& q_{i-1, \text { out }}(t) \\
& \quad=\min \left\{q_{m, i-1}, \rho_{i-1}(t) v_{f, i-1}, \frac{\rho_{i-1}(t) \lambda_{i} l_{i-1}}{T_{s}}\right\} .
\end{aligned}
$$

CF:

$$
q_{i-1, \text { out }}(t)=\min \left\{q_{m, i-1}, \frac{\rho_{i-1}(t) \lambda_{i} l_{i-1}}{T_{s}}\right\}
$$

\section{FC/CC:}

$$
q_{i-1, \text { out }}(t)=\max \left\{\min \left\{q_{m, i-1},\left[\rho_{J, i}(t)-\rho_{i}(t)\right] w_{i}-R_{i}(t), \frac{\rho_{i-1}(t) \lambda_{i} l_{i-1}}{T_{s}}\right\}, 0\right\} .
$$

According to the conservation law at the boundary of cell $i-1$ and cell $i$, we get

$$
q_{i, \text { in }}(t)=q_{i-1, \text { out }}(t)+R_{i}(t)
$$

where $R_{i}(t)$ is the on-ramp flow and $\rho_{J, i}(t)$ is the jam density of cell $i$.

In this paper, we extend the above simple state switch transmission rule to depict more complex cloverleaf junctions. As shown in Figure 2, each ramp is considered as a cell and the other types of junctions can be formulated in the simplified expression. The traffic flow transmission matrix can be denoted as the structure of (5). Here, we mainly consider the properties of cell $i_{k}+2(k=1,2,3,4)$ and rampcells by the following rules:

$$
\left\{\begin{array}{cccccc}
\text { Cell } & i_{k} & i_{k}+1 & i_{k}+1 & i_{k}+1 & i_{k}+1 \\
i_{k} & 0 & q_{i_{k}, i_{k}+1} & & & \\
i_{k}+1 & 0 & q_{i_{k}, i_{k}+1} & & \\
i_{k}+2 & & 0 & q_{i_{k}, i_{k}+1} & \\
i_{k}+3 & & & 0 & q_{i_{k}, i_{k}+1} \\
i_{k}+4 & & & & 0
\end{array}\right\}
$$

$$
k \in\{1,2,3,4\} \text {, }
$$

$$
\begin{array}{r}
\left\{\begin{array}{ccccc}
\text { Cell } & i_{k+1}+2 & i_{k-1}+4 & R_{r i_{k}} & R_{l i_{k}} \\
i_{k} & 0 & 0 & q_{i_{k}, R_{r i_{k}}} & 0 \\
i_{k}+2 & 0 & 0 & 0 & q_{i_{k}+2, R_{l i_{k}}} \\
R_{r i_{k}} & 0 & q_{R_{r i_{k}}, i_{k-1}+4} & 0 & 0 \\
R_{l i_{k}} & q_{R_{l i_{k}}, i_{k+1}+2} & 0 & 0 & 0
\end{array}\right\}, \\
\end{array}
$$

where

$$
\begin{aligned}
& q_{i_{k}+1, \text { in }}(t)=q_{i_{k}, i_{k}+1}(t), \\
& q_{i_{k}+2, \text { in }}(t)=q_{i_{k}+1, i_{k}+2}(t)+q_{R_{l_{k-1},}, i_{k}+2}(t), \\
& q_{i_{k}+3, \text { in }}(t)=q_{i_{k}+2, i_{k}+3}(t), \\
& q_{i_{k}+4, \text { in }}(t)=q_{i_{k}+3, i_{k}+4}(t)+q_{R_{r i_{k+1}}, i_{k}+4}(t), \\
& q_{R_{r i_{k}}, \text { in }}(t)=q_{i_{k}, R_{r i_{k}}}(t), \\
& q_{R_{l_{k}}, \text { in }}(t)=q_{i_{k}+2, R_{l i_{k}}}(t) .
\end{aligned}
$$

Here, $R_{r i_{k}}$ and $R_{l i_{k}}$ represent the right/left turning ramp-cell, respectively.

2.2. Finite Difference of the Higher-Order Equation. In this paper, we applied the PDE model proposed in [7]. This PDE model replaces the density gradient term with the speed gradient term to consider drivers' anticipation as follows:

$$
\frac{d v(x, t)}{d t}=\frac{\partial v}{\partial t}+v \frac{\partial v}{\partial x}=\frac{v_{e}(\rho)-v}{T}+w \frac{\partial v}{\partial x}
$$

where $w$ represents the propagation speed of the disturbance. In this model, the characteristic speed is equal to or smaller than the macroscopic flow velocity. In reality, traffic density $\rho(x, t)$ is hard to investigate or estimated directly (Figure 8); the most popular method is to monitor the time occupancy of vehicles passing detection stations. A number of literatures indicate that the time occupancy at $x$ is proportional to traffic density around $x$.

Assuming there exist no merging or diverging in the section, say $g(x, t)=0$, and applying the finite difference method to discretize (1a) and (7), we obtain the following difference equations based on point detectors like inductive 


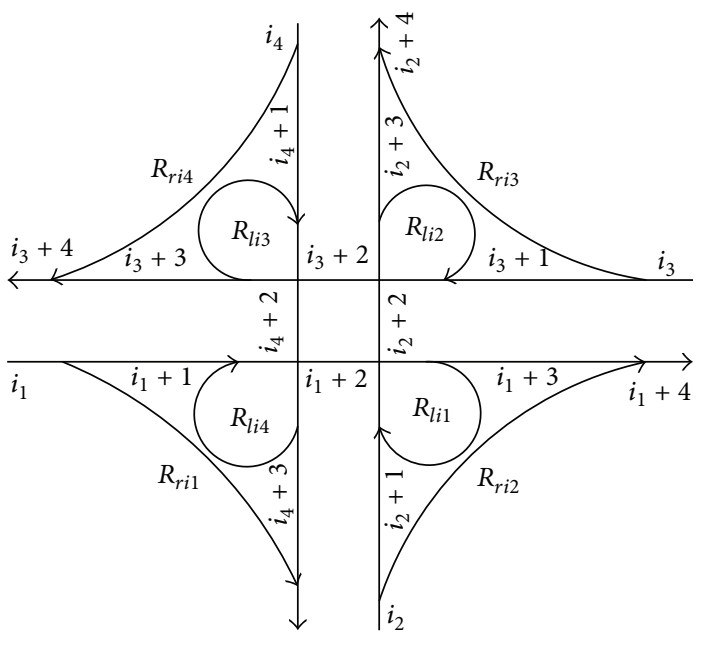

(a)

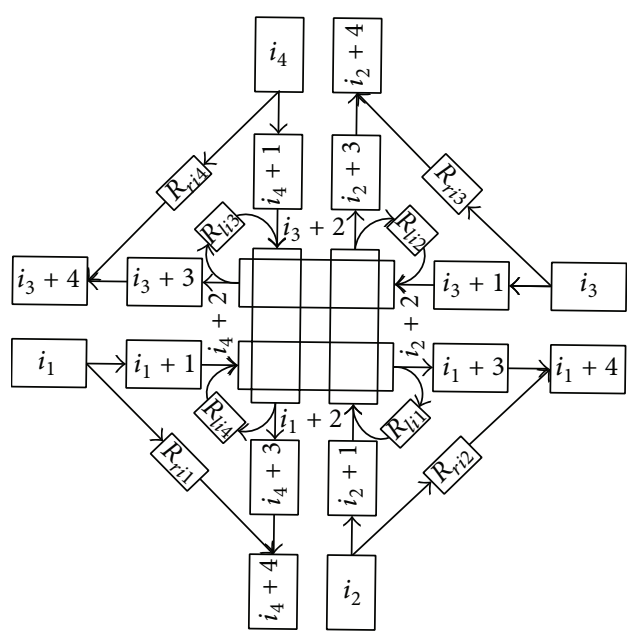

(b)

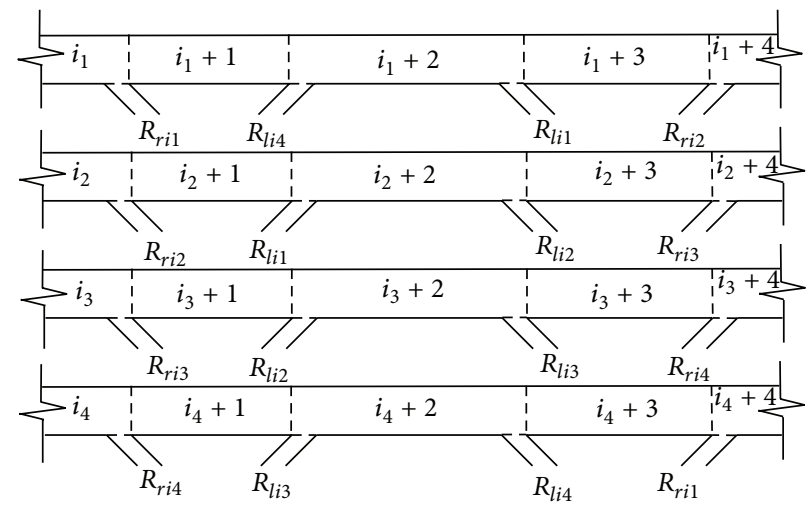

(c)

FIGURE 2: Cell representation of a cloverleaf junction: (a) the layout of junction; (b) the corresponding division of cells; (c) the corresponding CTM cells.

loops, microwave detectors, and radar sensors. The fundamental equation of traffic flow is

$$
q_{i}(t)=\frac{\lambda_{i} o_{i}(t) v_{i}(t)}{g_{i}(t)}+\zeta_{i}^{q}(t),
$$

where $o_{i}(t)$ denotes the time occupancy at the upstream boundary of cell $i, g_{i}(t)$ represents the effective vehicle length which depends on both the actual vehicle length, which varies by lane and over the course of a day, and the loop's electrical circuit, and $\zeta_{i}^{q}(t)$ is the zero-mean white noise acting on the approximation to reflect the measurement inaccuracies and fluctuations in the traffic flow.

During one time interval, the drivers in cell $i$ will adapt their speed to the local traffic density and are thus impacted by the traffic state in front of them. We suppose the adaptive behavior is influenced by the composition of traffic density of these two adjacent cells; it is a weighted average of the density in cell $i$ and in cell $i+1$ :

$$
\overline{o_{i}}(t)=f_{o}\left[\alpha_{i}(t), o_{i}(t), o_{i+1}(t)\right] .
$$

To simplify the combinational equations, we assume that this adaptation is linear as

$$
\overline{o_{i}}(t)=\alpha_{i} o_{i}(t)+\left(1-\alpha_{i}\right) o_{i+1}(t)
$$

where the parameter $0<\alpha_{i}<1$ weights how far ahead the drivers look and how well they anticipate the traffic density downstream. The drivers adapt the local traffic density more habitually; $\alpha_{i}$ is closer to 1 .

Since each drivers' aggressiveness is random, the anticipant average space speed of cell $i$ is relative to the speed of cell $i$ and cell $i+1$; the weighted factor $0<\beta_{i}<1$ expresses the relative impact on the calculation of the speed of convective and inertial behavior:

$$
\overline{v_{i}}(t)=f_{v}\left[\beta_{i}(t), v_{i}(t), v_{i+1}(t)\right]
$$

Similarly, we assume that

$$
\overline{v_{i}}(t)=\beta_{i} v_{i}(t)+\left(1-\beta_{i}\right) v_{i+1}(t) .
$$


Because most of drivers tend to adapt their behavior whose function reflects an deterministic statistical relationship between the traffic flow and density or occupancy; the anticipant traffic flow $y_{i, \text { out }}(t)$ that passes through the boundary of cell $i$ and cell $i+1$ is approximately equal to $o_{i}(t) \overline{v_{i}}(t)$, when the stationary equivalent traffic flow is taken into consideration.

Meanwhile, the transmission flow is also constrained by the current sending capacity of cell $i$, that is, $o_{i}(t) l_{i} / T_{s}$, and by the receiving capacity of cell $i+1$, that is, $\left[o_{m}-o_{i}(t)\right] l_{i+1} / T_{s}$, where $o_{m}$ is the maximum occupancy; then it yields

$$
\begin{aligned}
& y_{i, \text { out }}(t) \\
& \quad=\min \left\{\frac{o_{i}(t) l_{i}}{T_{s}}, \frac{\left[o_{m}-o_{i+1}(t)\right] l_{i+1}}{T_{s}}, \gamma_{i} o_{i}(t) \overline{v_{i}}(t)\right. \\
& \left.\quad+\left(1-\gamma_{i}\right) Q_{e}\left[\overline{o_{i}}(t)\right]\right\}+\zeta_{i}^{y}(t),
\end{aligned}
$$

where $Q_{e}\left[\overline{o_{i}}(t)\right]$ is the stationary equivalent traffic flow and time occupancy relationship function, $Q_{e}\left[\overline{o_{i}}(t)\right]=Q_{e 1}\left[\overline{o_{i}}(t)\right]$, if $\overline{o_{i}}(t)<o_{c, i}(t) ; Q_{e}\left[\overline{o_{i}}(t)\right]=Q_{e 1}\left[\overline{o_{i}}(t)\right]$, otherwise. $o_{c, i}(t)$ is the critical time occupancy of cell $i$, and the coefficient $0<$ $\gamma_{i}<1$ expresses the relative impact on the calculation of the equivalent traffic flow of convective and macroscopic stable behavior and $\zeta_{i}^{y}(t)$ is a zero-mean white noise that describes the uncertainty of traffic transmission between two adjacent cells.

Thus, we can formulate the evolution of time occupancy under the assumption that $g_{i}(t)$ is approximately equal to $g_{i+1}(t)$ by the following finite difference equation:

$$
o_{i}(t+1)=o_{i}(t)+\frac{T_{s}}{\lambda_{i} l_{i}}\left[y_{i, \text { in }}(t)-y_{i, \text { out }}(t)\right],
$$

where $y_{i, \text { in }}(t)$ is equal to $y_{i, \text { out }}(t)$ when there exist no on/offramps. The formulas of conditions with merging or diverging areas can be obtained similarly as

$$
v_{i}(t+1)= \begin{cases}v_{i}(t)+\frac{T_{s}}{l_{i}}\left[w_{i}-v_{i}(t)\right]\left[v_{i+1}(t)-v_{i}(t)\right]+\frac{T_{s}}{T}\left[V_{e}\left(o_{i}(t)\right)-v_{i}(t)\right]+\zeta_{i}^{v}(t), & \text { if } v_{i}(t)<w_{i} \\ v_{i}(t)+\frac{T_{s}}{l_{i}}\left[w_{i}-v_{i}(t)\right]\left[v_{i}(t)-v_{i-1}(t)\right]+\frac{T_{s}}{T}\left[V_{e}\left(o_{i}(t)\right)-v_{i}(t)\right]+\zeta_{i}^{v}(t), & \text { if } v_{i}(t) \geq w_{i},\end{cases}
$$

where $w_{i}$ represents the propagation speed of the disturbance that is the backward speed of traffic shockwave and $\zeta_{i}^{v}(t)$ is a zero-mean white noise reflecting the fluctuations in the drivers' speed.

\section{Simulation Results}

In the following experiments, we apply the initial stationary equivalent traffic speed and occupancy relationship function as (15) and the corresponding traffic flow and time occupancy relationship function as (16) given in [10]:

$$
\begin{aligned}
V_{e}\left[\overline{o_{i}}(t)\right] & =v_{f} \exp \left[-\frac{1}{\alpha}\left(\frac{\overline{o_{i}}(t)}{o_{c, i}(t)}\right)^{\alpha}\right], \\
Q_{e}\left[\overline{o_{i}}(t)\right] & =\overline{o_{i}}(t) V_{e}\left[\overline{o_{i}}(t)\right] \\
& =v_{f} \overline{o_{i}}(t) \exp \left[-\frac{1}{\alpha_{m}}\left(\frac{\overline{o_{i}}(t)}{o_{c, i}(t)}\right)^{\alpha_{m}}\right] .
\end{aligned}
$$

In the simulation of traffic density and speed evolution of a segment of urban freeways with periodical boundary conditions, the following constants that keep the same in all cells are applied. Geometric conditions and temporal-spatial discretization are

$$
\begin{aligned}
L & =10 \mathrm{~km}, \\
\Delta L & =0.1 \mathrm{~km}, \\
T_{s} & =1 \mathrm{~s},
\end{aligned}
$$

$$
\begin{gathered}
T=10 \mathrm{~s}, \\
g(x, t)=6.67 \mathrm{~m} .
\end{gathered}
$$

Coefficients of fundamental diagram are

$$
\begin{aligned}
v_{f} & =80 \mathrm{~km} / \mathrm{h}, \\
w & =30 \mathrm{~km} / \mathrm{h}, \\
o_{c, i}(t) & =20 \%, \\
Q_{m} & =1800 \mathrm{veh} / \mathrm{h} / \text { lane. }
\end{aligned}
$$

Parameters of drivers' adaptive behavior and standard deviations of Gaussian noise are

$$
\begin{aligned}
\alpha_{i}(t) & =0.5, \\
\beta_{i}(t) & =0.5, \\
\gamma_{i}(t) & =0.5, \\
\sigma\left(\zeta_{i}^{v}(t)\right) & =\sigma\left(\zeta_{i}^{q}(t)\right)=0 .
\end{aligned}
$$

Generally, the adaptive time can be chosen as 0 to 1 . For numerical tests shown in this section, we just choose adaptive coefficients as 0.5 to show the influence of drivers' adaptive behavior.

3.1. Experiment 1: Linear Initialization of Density. In Experiment 1 , we test the influence of linear initialization of density (Figure 5). During the whole process of simulation, lane 


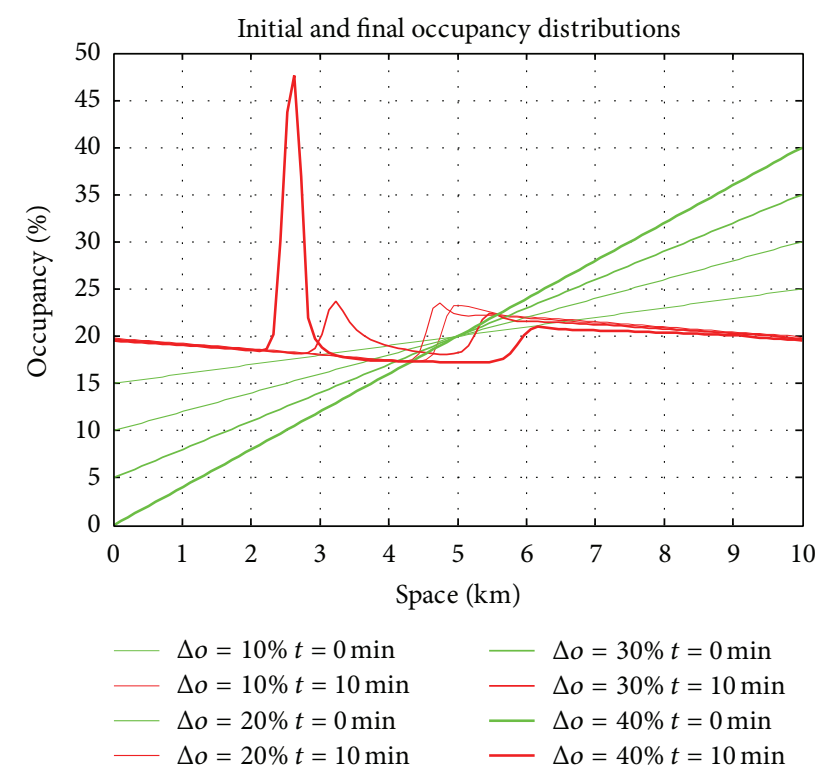

FIGURE 3: The initial and finial spatial distributions of occupancy for Experiment 1.

number and speed limit (equal to free-flow speed $v_{f}$ ) are not changed. Four typical experiments are conducted with the same critical occupancy of $20 \%$; the boundary occupancy conditions are $[15 \%, 25 \%],[10 \%, 30 \%],[5 \%, 35 \%]$, and $[0 \%$, $40 \%]$, respectively. Therefore, the initial differences between critical occupancy are 5\%, 10\%, 15\%, and $20 \%$.

The results show that a larger initial swing leads to a more obvious backward moving jam that is observed in Figure 3. Waves moving in the opposite directions meet at around $3.5 \mathrm{~min}$ and evolve into the backward moving jam with a larger density and a smaller fluctuation that propagates downstream especially obtained in the last experiment. Similarly, the evolution pattern of traffic speed shows the wave fluctuation phenomena of a moving jam in Figure 4.

When the initial density swing is small, the two reverse waves meet and the gap arises but no moving jam is achieved because of the lack of enough density difference between adjacent cells where the fluctuations cross. The scatter plot of fundamental diagram is illustrated in Figure 6; the widest line represents the equivalent functions of traffic speed/occupancy and traffic flow/occupancy with exponential expression showed in (16). Points of four different colors are four experimental results, respectively. It indicates that a linear initialization with a larger swing is more nonstable and the results scatter in a larger area in the fundamental diagram. The numerical results indicate that a larger initial difference of density generally triggers a larger fluctuation of traffic flow.

3.2. Experiment 2: Local Cluster Effect. The local cluster effect corresponds to the stop-and-go phenomena observed in the field due to a small disturbance [7]. In this subsection, we simulate the local cluster effect with respect to a localized perturbation in an initial homogeneous condition.
The following initial variation of the average occupancy $o_{0}$ is used as in [17]:

$$
\begin{aligned}
& o(x, 0)=o_{0}+\Delta o_{0}\left\{\cosh ^{-2}\left[\frac{160}{L}\left(x-\frac{3 L}{8}\right)\right]\right. \\
& \left.-\frac{1}{4} \cosh ^{-2}\left[\frac{40}{L}\left(x-\frac{13 L}{32}\right)\right]\right\},
\end{aligned}
$$

where $v(x, 0)=V_{e}[o(x, 0)]$; the other parameters are the same as the simulation of linear initialization and the second term acts as a local perturbation to the unstable constant distribution; then the combinatorial difference methods are used to show the evolution pattern when $o(x, 0)$ increases.

Figure 7 shows the initial local perturbations with $\Delta o_{0}=$ $5 \%$ eventually evolve into local cluster effects when initial distribution of average time occupancy is limited in a specific extent and periodic boundary conditions are applied. The observed local cluster effects are also consistent with the diverse nonlinear dynamics phenomena observed in realistic traffic flow.

\section{Model Validation and Application}

The real data testing was conducted with traffic measurements collected from a $4.7 \mathrm{~km}$, 3-lane stretch of Queen Elizabeth Way, Canada. As shown in Figure 9, it involves two on-ramps and an off-ramp at stations 50 and 53; another five loop detector stations were installed along this freeway and three at the on/off-ramps. The test stretch is subdivided into 8 segments, each with an approximate length of $650 \mathrm{~m}$. Furthermore, a more refined cell division (where the size is only about $125 \mathrm{~m}$ ) is conducted to explore the detailed spatialtemporal evolution of traffic jam.

The individual vehicle data were recorded by the detectors from 6 am to $10 \mathrm{am}$ on December 15, 1998. All data were converted into aggregated traffic measurements of flow and space mean speed with a temporal-resolution-level 20 seconds. Only traffic flow and occupancy data are used for validation.

For the sake of simple and convenient computation, we use piecewise linear assumption of fundamental diagram in this section:

$$
\begin{aligned}
& Q_{e}[o(x, t)]=v_{f}(x, t) o(x, t) \\
& \text { if } o(x, t)<o_{c}(x, t) ; \\
& Q_{e}[o(x, t)]=w(x, t)\left[o_{J}(x, t)-o(x, t)\right], \\
& \text { otherwise. }
\end{aligned}
$$

Figure 10 shows the fundamental diagram defined in this paper, where points A and B are the critical milestone of the formation and dissipation of traffic congestion; the corresponding occupancies are $k_{q}$ and $k_{d}$ where " $q$ " means queuing and " $d$ " means dissipating. Regressive results of the real traffic data show the occupancy gap is accepted that midpoint of line $\mathrm{AB}$ is the inflexion of traffic states. As a result, the assumption of critical occupancy is approximately to $k_{c}$. So, we determine that we meet a congested state if and only 

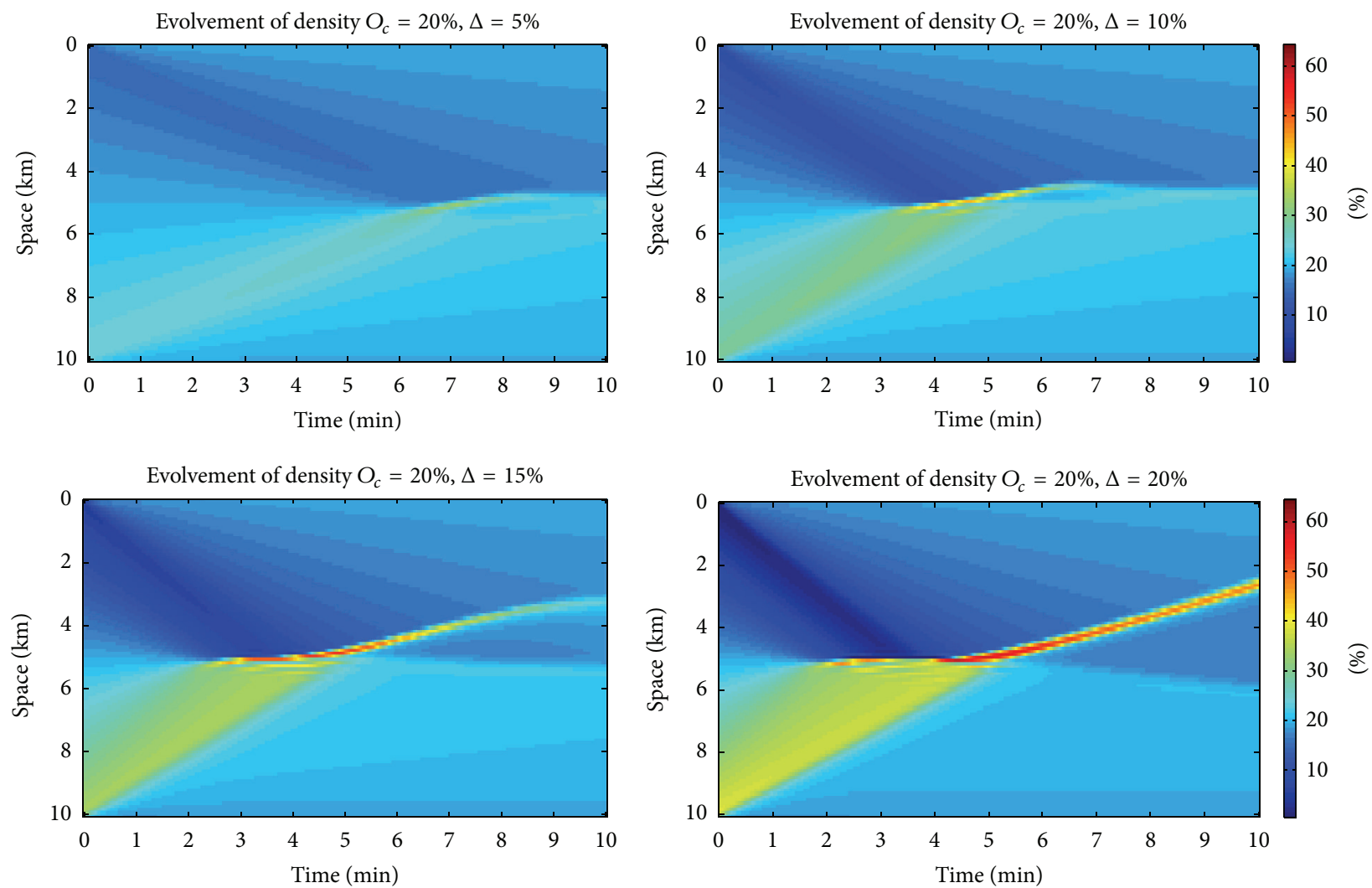

FIgURE 4: Occupancy evolution under linear initialization for Experiment 1.
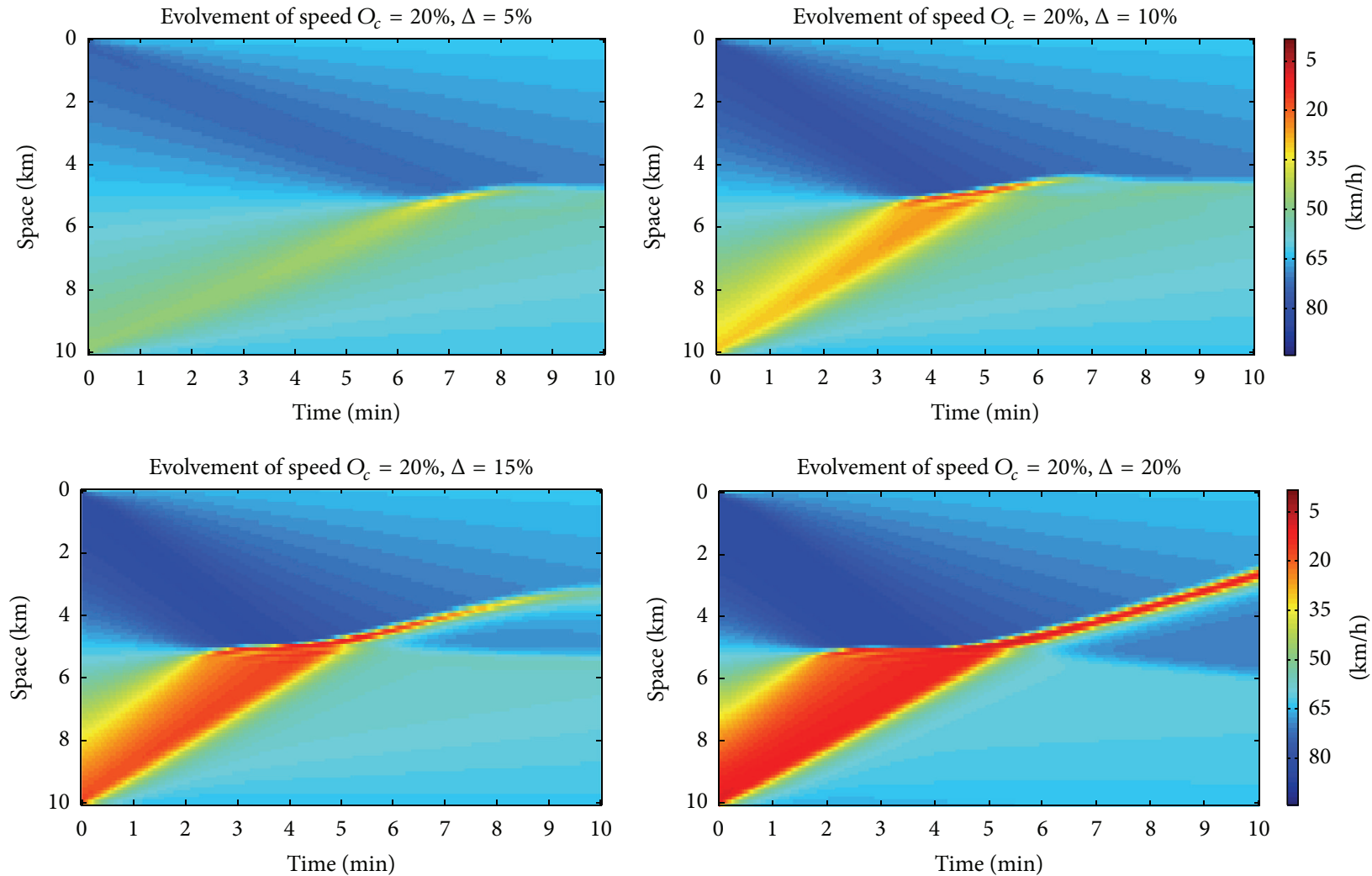

FIgURE 5: Speed evolution under linear initialization for Experiment 1. 

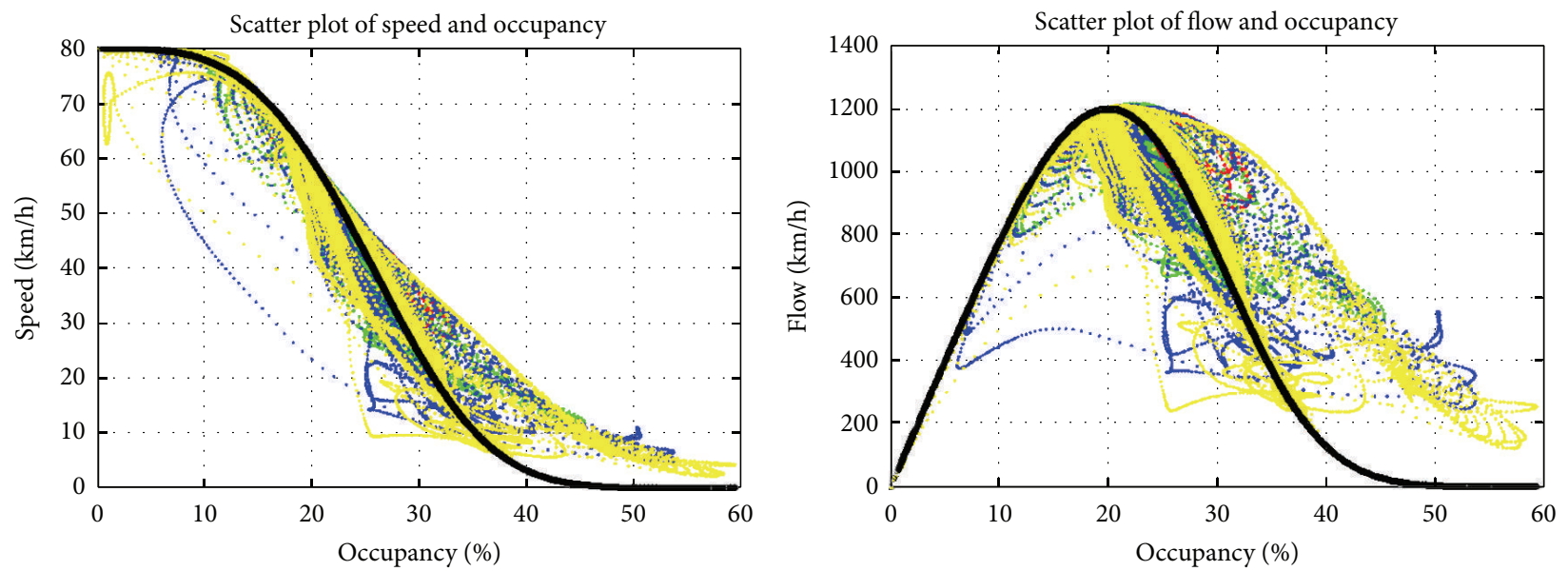

Figure 6: Fundamental diagram obtained for Experiment 1.

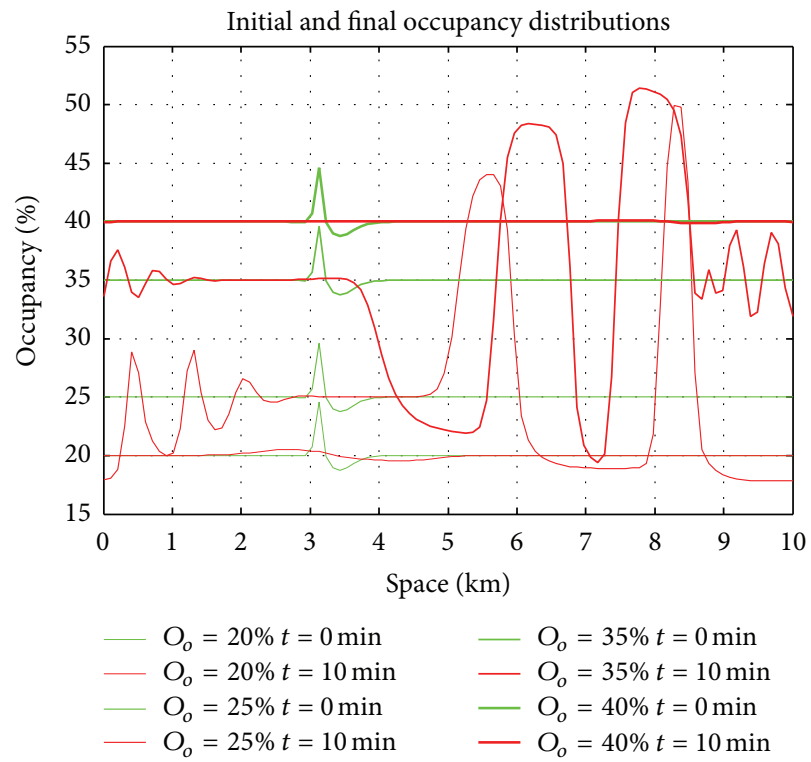

FIGURE 7: The initial and finial spatial distributions of occupancy for Experiment 2.

if the $o(x, t)>o_{c}\left(\right.$ or $\left.k_{c}\right)$ and $q(x, t) / o(x, t)<\left[q_{q}(x, t)-\right.$ $\left.q_{d}(x, t)\right] /\left[o_{q}(x, t)-o_{d}(x, t)\right]$.

It is supposed that the traffic data collected by station 49 52 is unknown, and only date from stations 48 and 53 and the on/off-ramps is used to validate the finite different method discussed in this paper. The comparative results of measured and simulated occupancy data are shown in Figure 11. The agreement between two kinds of data suggests that this finite difference method is well applied to reflect the traffic congestion and the influences of on/off-ramp.

Tests also show that the simulated results will significantly deviate from the empirical measurements, if no drivers' anticipation term is introduced in the traffic flow model.

\section{Conclusive Remarks}

An improved spatial-temporal finite difference method based on LWR and a higher-order car-following model is proposed. A compositional model combining cell transmission model (CTM) and drivers' adaptive car-following behaviors is proposed for the spatial-temporal evolution of traffic flows on freeways.

Numerical tests verify that the model is able to simulate complex traffic phenomena observed in the field such as shock waves, stop-and-go waves, and local cluster effects. Empirical traffic data are also used to validate the finite difference method proposed in this paper. The results are 


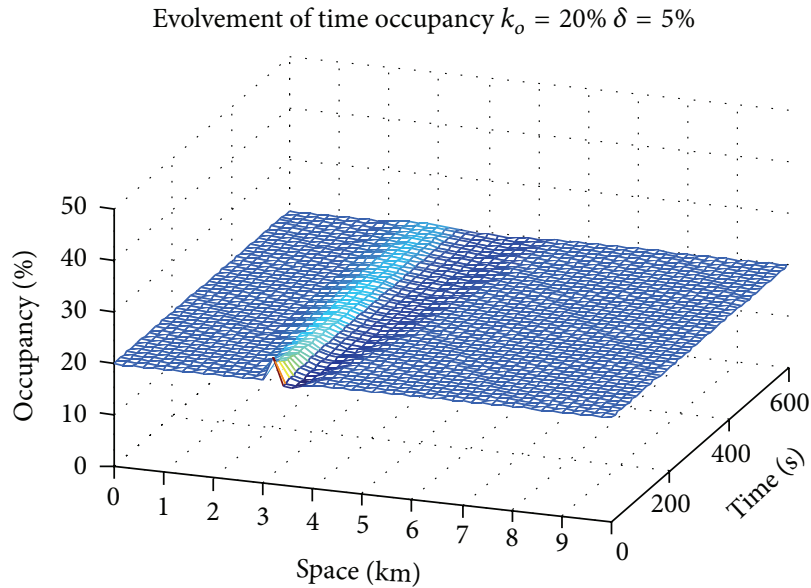

Evolvement of time occupancy $k_{o}=35 \% \delta=5 \%$

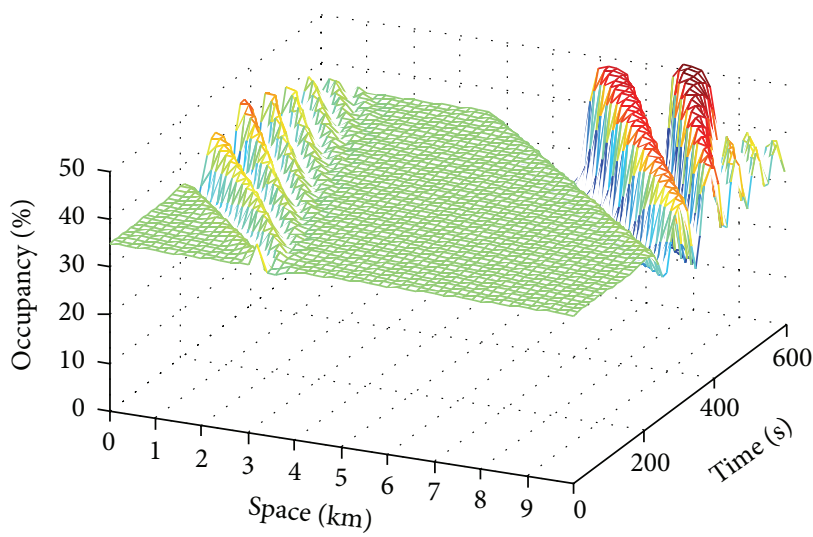

Evolvement of time occupancy $k_{o}=25 \% \delta=5 \%$

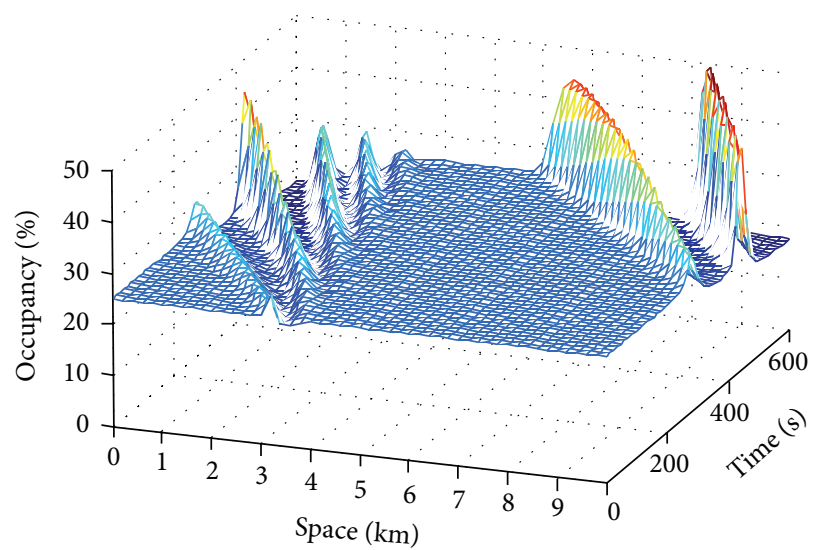

Evolvement of time occupancy $k_{o}=40 \% \delta=5 \%$

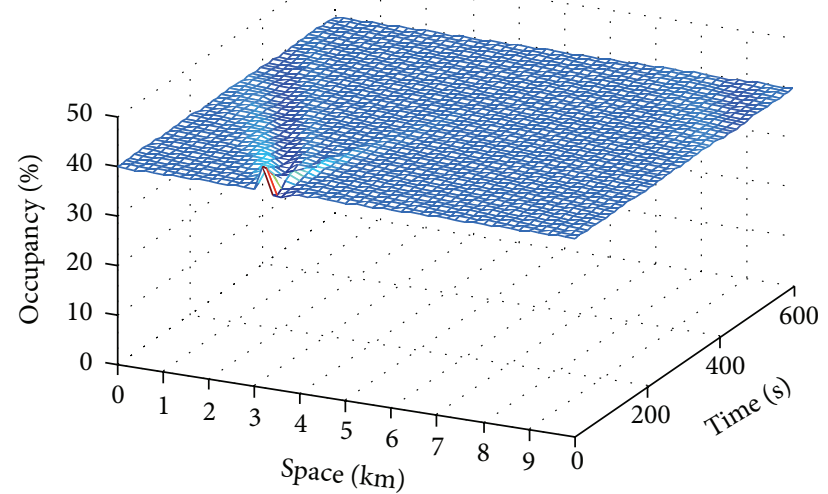

FIGURE 8: Traffic density evolution under small local disturbance for Experiment 2.

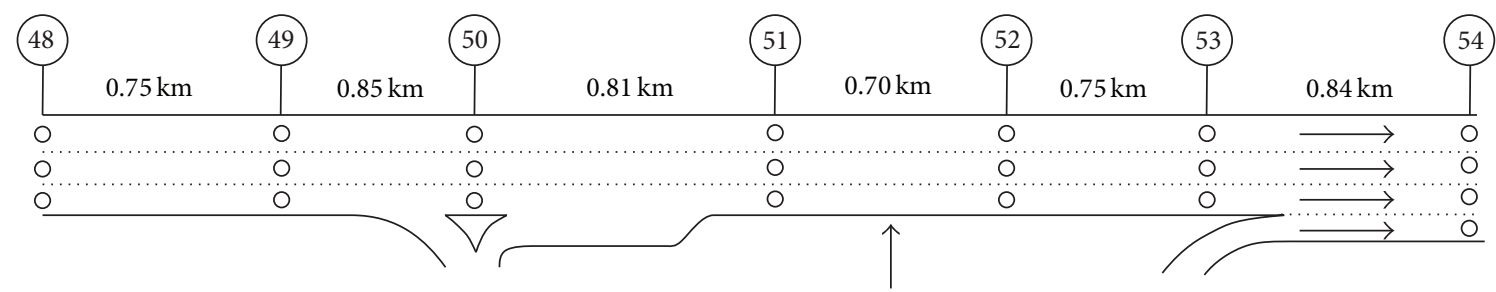

$\begin{array}{lll}\text { Match line Cawthra Road } & \text { Boottleneck } & \text { East of Dixie Road }\end{array}$
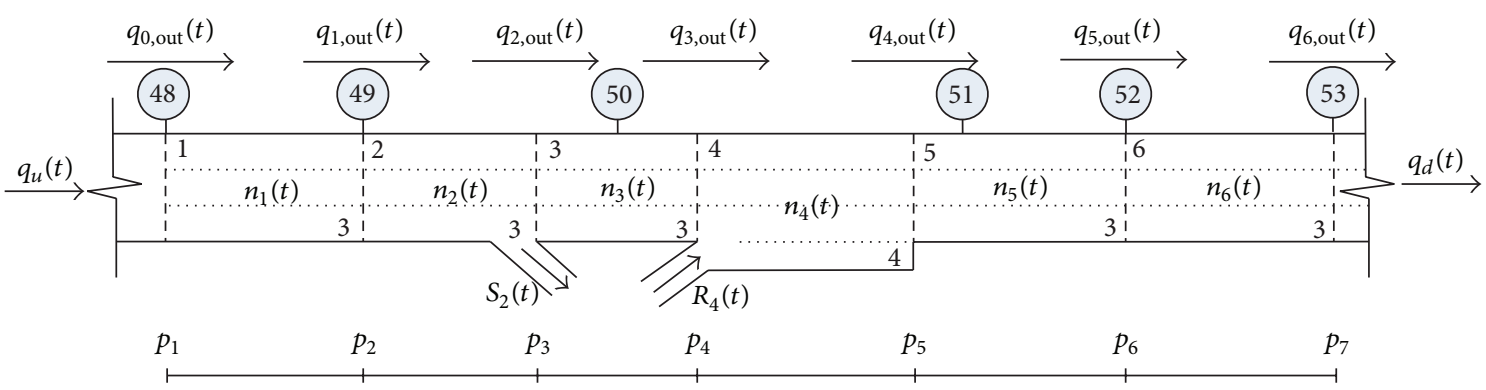

FIGURE 9: The layout and the corresponding cell representation of Queen Elizabeth Way, Canada. 


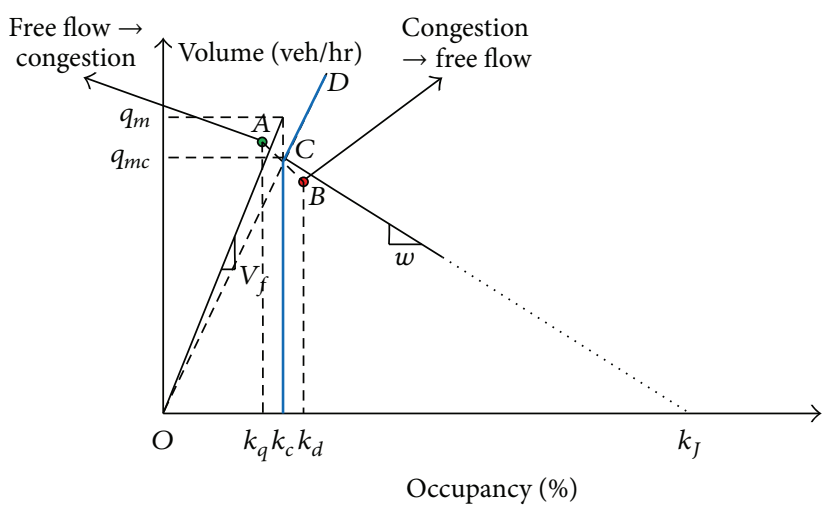

Figure 10: The fundamental diagram defined to depict asymmetric formation and dissipation of traffic congestion.
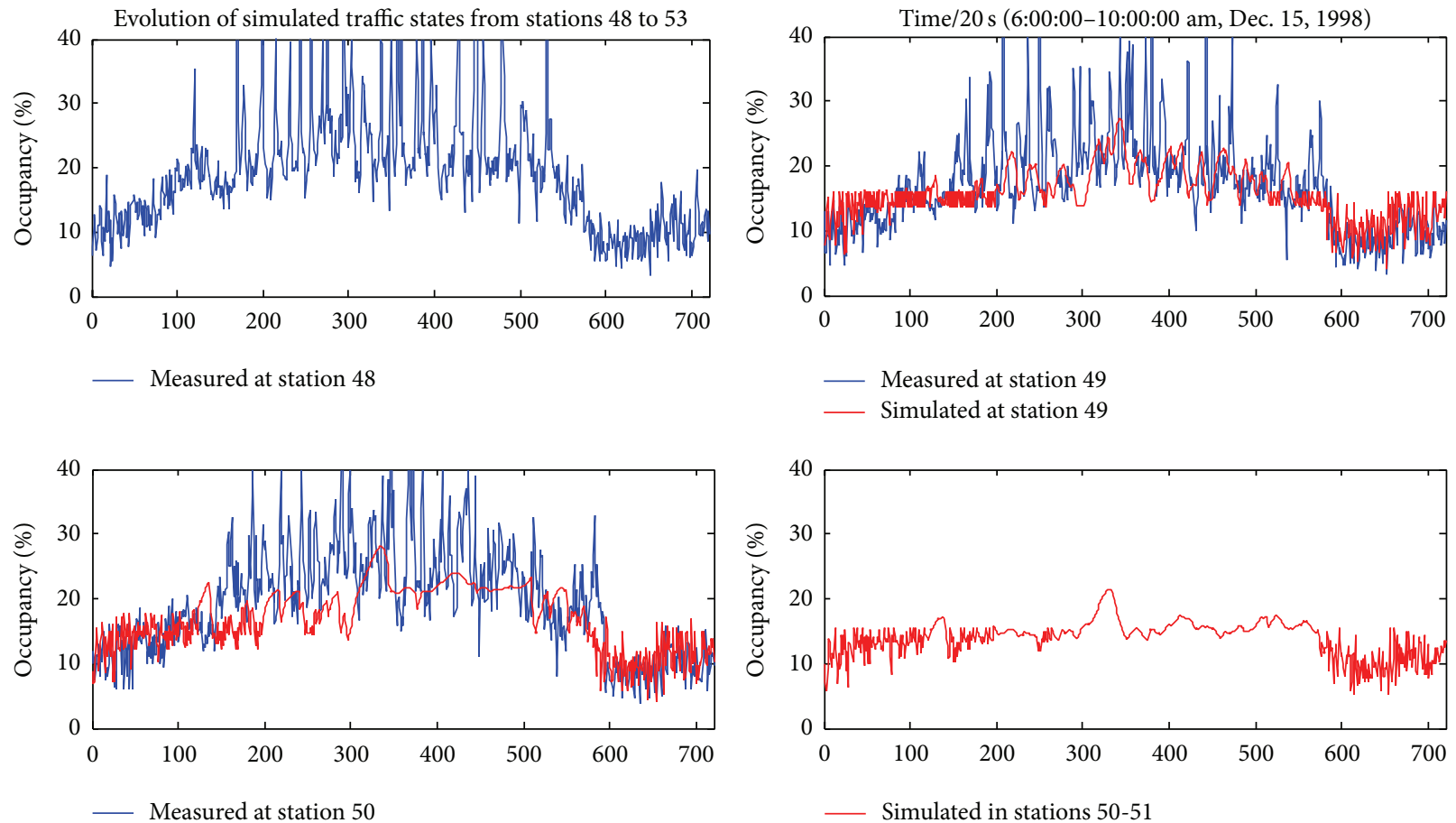

- Simulated at station 50
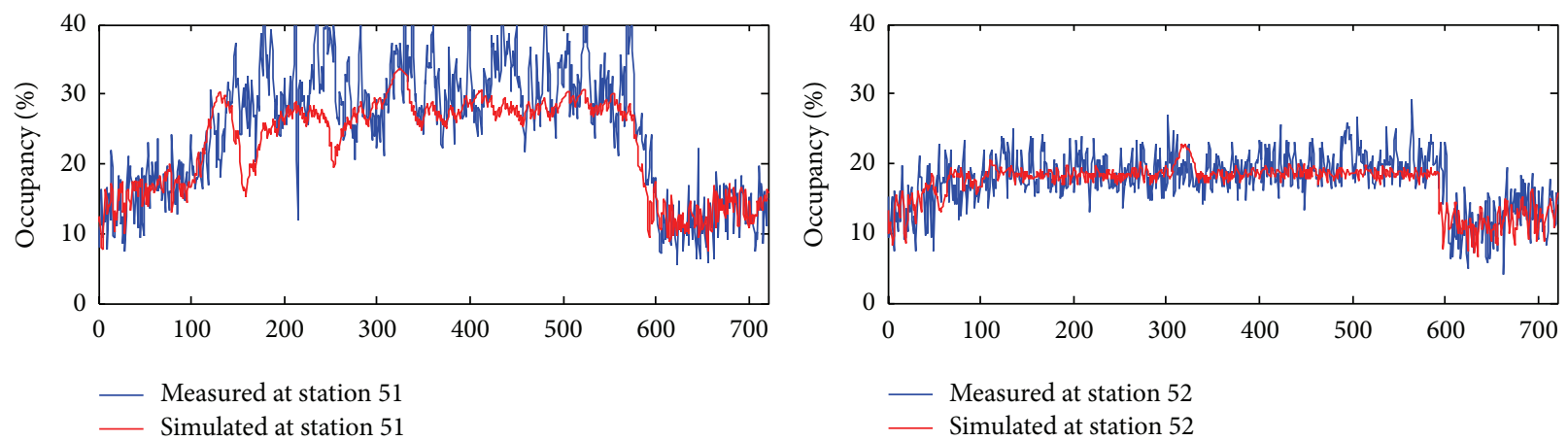

FIGURE 11: Spatial-temporal evolution of empirical and simulated occupancy. 
consistent with the nonlinear dynamic properties measured in practice.

\section{Conflict of Interests}

The authors declare that there is no conflict of interests regarding the publication of this paper.

\section{Acknowledgment}

The supports of the National Natural Science Foundation of China (71303269), the Fundamental Research Funds for the Central Universities, Central University of Finance and Economics Major Research Task of Fostering Project (14ZZD006) are gratefully acknowledged.

\section{References}

[1] M. J. Lighthill and G. B. Whitham, "On kinematic waves. II. A theory of traffic flow on long crowded roads," Proceedings of the Royal Society of London Series A: Mathematical and Physical Sciences, vol. 229, no. 1178, pp. 317-345, 1955.

[2] P. I. Richards, "Shock waves on the highway," Operations Research, vol. 4, pp. 42-51, 1956.

[3] C. F. Daganzo, "The cell transmission model: a dynamic representation of highway traffic consistent with the hydrodynamic theory," Transportation Research Part B: Methodological, vol. 28, no. 4, pp. 269-287, 1994.

[4] C. F. Daganzo, "A finite difference approximation of the kinematic wave model of traffic flow," Transportation Research Part $B$, vol. 29, no. 4, pp. 261-276, 1995.

[5] R. Horowitz, X. T. Sun, L. Muñoz et al., "Design, field implementation and evaluation of adaptive ramp metering algorithms: final report," California PATH Research Report UCB-ITS-PRR2006-21, 2006.

[6] H. K. Lo, E. Chang, and Y. C. Chan, "Dynamic network traffic control," Transportation Research Part A: Policy and Practice, vol. 35, no. 8, pp. 721-744, 2001.

[7] R. Jiang, Q.-S. Wu, and Z.-J. Zhu, "A new continuum model for traffic flow and numerical tests," Transportation Research Part B: Methodological, vol. 36, no. 5, pp. 405-419, 2002.

[8] H. J. Payne, "Models of freeway traffic and control," in Mathematical Models of Public Systems, G. A. Bekey, Ed., vol. 1 of Simulation Council Proceedings Series, pp. 51-61, 1971.

[9] A. S. Willsky, E. Y. Chow, S. B. Gershwin, C. S. Greene, A. L. Kurkjian, and P. K. Houpt, "Dynamic model-based techniques for the detection of incidents on freeways," IEEE Transactions on Automatic Control, vol. 25, no. 3, pp. 347-360, 1980.

[10] M. Papageorgiou, J.-M. Blosseville, and H. Hadj-Salem, "Modelling and real-time control of traffic flow on the southern part of Boulevard Périphérique in Paris: Part I: modelling," Transportation Research Part A: General, vol. 24, no. 5, pp. 345359, 1990.

[11] A. Kotsialos, M. Papageorgiou, C. Diakaki, Y. Pavlis, and F. Middelham, "Traffic flow modeling of large-scale motorway networks using the macroscopic modeling tool METANET," IEEE Transactions on Intelligent Transportation Systems, vol. 3, no. 4, pp. 282-292, 2002.
[12] Y. Wang, M. Papageorgiou, and A. Messmer, "Real-time freeway traffic state estimation based on extended Kalman filter: adaptive capabilities and real data testing," Transportation Research Part A: Policy and Practice, vol. 42, no. 10, pp. 1340-1358, 2008.

[13] C. D. Alecsandru, A stochastic mesoscopic cell-transmission model for operational analysis of large-scale transportation networks [Ph.D. thesis], Louisiana State University, Baton Rouge, La, USA, 2006.

[14] Y. D. Lu, S. C. Wong, M. P. Zhang, C.-W. Shu, and W. Q. Chen, "Explicit construction of entropy solutions for the Lighthill-Whitham-Richards traffic flow model with a piecewise quadratic flow-density relationship," Transportation Research Part B: Methodological, vol. 42, no. 4, pp. 355-372, 2008.

[15] T. L. Pan, A. Sumalee, R. X. Zhong, and N. Indra-Payoong, "Short-term traffic state prediction based on temporal-spatial correlation," IEEE Transactions on Intelligent Transportation Systems, vol. 14, no. 3, pp. 1242-1254, 2013.

[16] R. X. Zhong, A. Sumalee, T. L. Pan, and W. H. K. Lam, "Stochastic cell transmission model for traffic network with demand and supply uncertainties," Transportmetrica A: Transport Science, vol. 9, no. 7, pp. 567-602, 2013.

[17] M. Herrmann and B. S. Kerner, "Local cluster effect in different traffic flow models," Physica A: Statistical Mechanics and Its Applications, vol. 255, no. 1-2, pp. 163-188, 1998. 


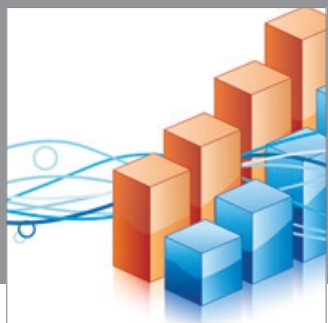

Advances in

Operations Research

mansans

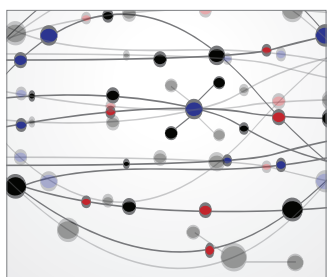

The Scientific World Journal
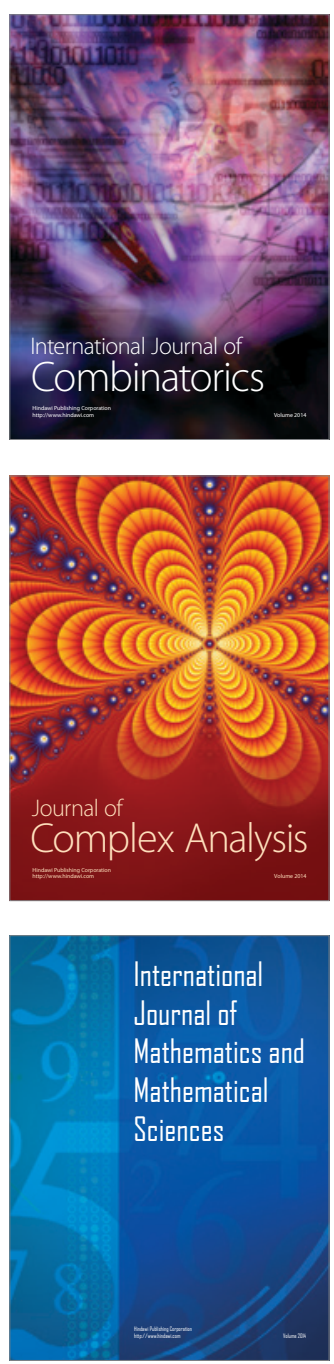
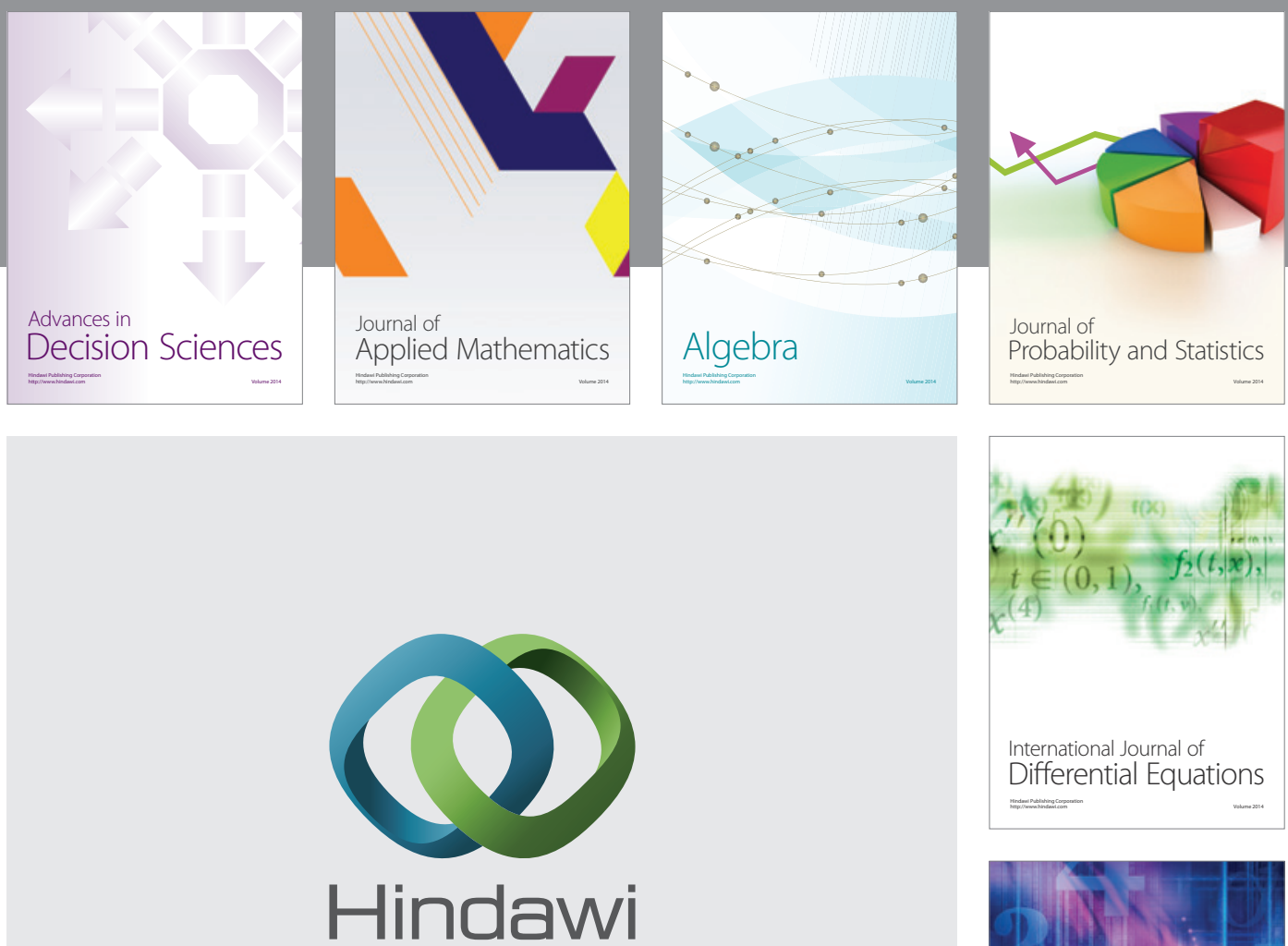

Submit your manuscripts at http://www.hindawi.com
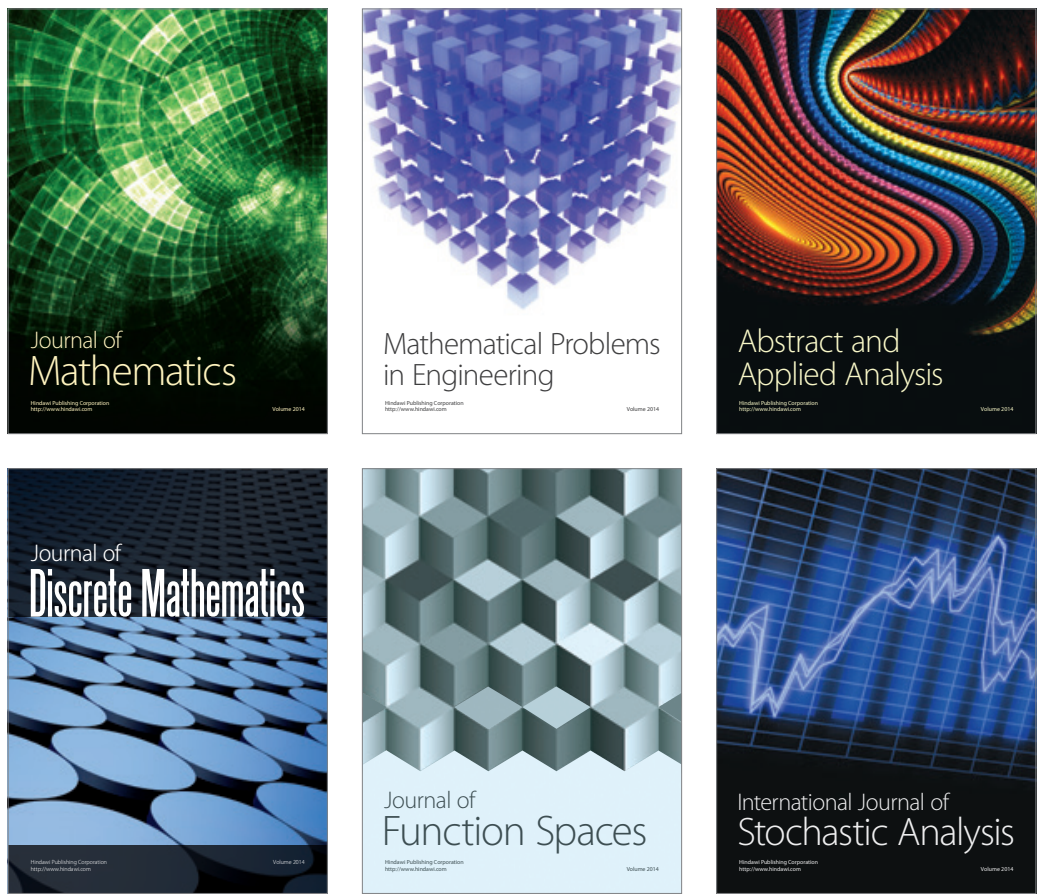

Journal of

Function Spaces

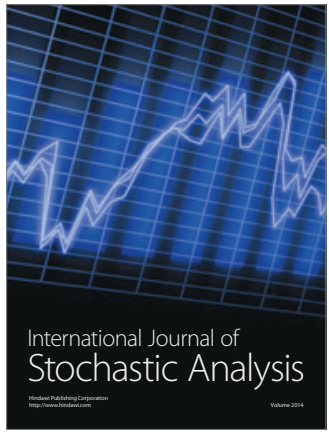

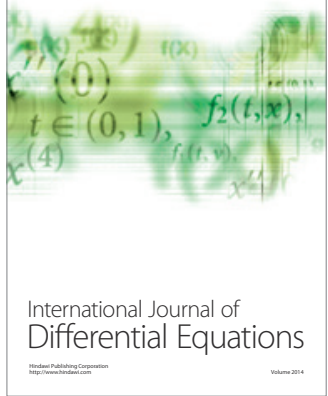
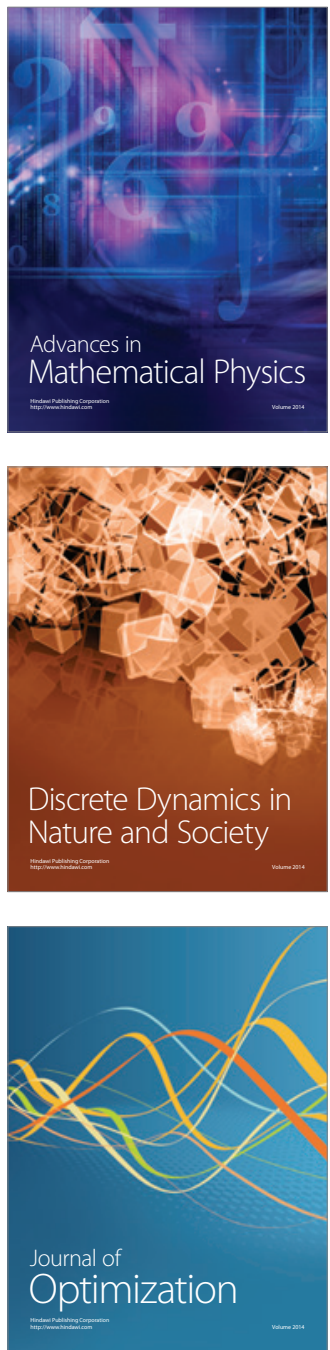